\title{
Massless propagators: applications in QCD and QED
}

\section{P. A. Baikov}

Institute of Nuclear Physics, Moscow State University, Moscow 119899, Russia

E-mail: baikov@theory.sinp.msu.ru

\section{K. G. Chetyrkin*}

Institut für Theoretische Teilchenphysik, Universität Karlsruhe, D-76128 Karlsruhe, Germany

E-mail: cheteparticle.uni-karlsruhe.de

\section{J. H. Kühn}

Institut für Theoretische Teilchenphysik, Universität Karlsruhe, D-76128 Karlsruhe, Germany

E-mail: jkeparticle.uni-karlsruhe.de

We report on two recent results based on the evaluation of five-loop massless propagators in QCD and QED: (i) corrections of order $\alpha_{s}^{4}$ to the absorptive part of the polarization function in QCD with $n_{f}=3$; (ii) the five-loop contribution to the $\beta$ function of quenched QED.

8th International Symposium on Radiative Corrections (RADCOR)

October 1-5 2007

Florence, Italy

${ }^{*}$ Speaker. 


\section{Introduction}

Propagators - that is Feynman Integrals depending on only one external momenta - appeared in QFT from its very beginning, for instance, in the description of the vacuum polarization in QED and since then constitute an important set of Feynman integrals to deal with.

Massless propagators are an indispensable tool in Renormalization Group (RG) calculations within the framework of Dimensional Regularization [1] and the so-called Minimal Subtractions schemes [2].

Massless propagators appear in many important physical applications. The total cross-section of $e^{+} e^{-}$annihilation into hadrons, the Higgs decay rate into hadrons, the semihadronic decay rate of the $\tau$ lepton, the running of the fine structure coupling are all computable in the high energy limit in terms of massless propagators.

The strong coupling constant $\alpha_{s}$ is one of the three fundamental gauge couplings constants of the Standard Model of particle physics. Its precise determination is one of the most important aims of particle physics. One of the most precise and theoretically safe determination of $\alpha_{s}$ is based on measurements of the cross section for electron-positron annihilation into hadrons. These have been performed in the low-energy region between $2 \mathrm{GeV}$ and $10 \mathrm{GeV}$ and, in particular, at and around the $Z$ resonance at $91.2 \mathrm{GeV}$. Conceptually closely related is the measurement of the semileptonic decay rate of the $\tau$-lepton, leading to a determination of $\alpha_{s}$ at a scale below $2 \mathrm{GeV}$.

From the theoretical side, in the framework of perturbative QCD, these rates and cross sections are evaluated as inclusive rates into massless quarks and gluons [3, 4]. (Power suppressed mass effects are well under control for $e^{+} e^{-}$-annihilation, both at low energies and around the $Z$ resonance, and for $\tau$ decays $[5,6,7,8,9,10]$, and the same applies to mixed QCD and electroweak corrections $[11,12])$.

The ratio $R(s) \equiv \sigma\left(e^{+} e^{-} \rightarrow\right.$ hadrons $) / \sigma\left(e^{+} e^{-} \rightarrow \mu^{+} \mu^{-}\right)$is expressed through the absorptive part of the correlator

$$
\Pi_{\mu v}(q)=i \int \mathrm{d} x e^{i q x}\left\langle 0\left|T\left[j_{\mu}^{\mathrm{em}}(x) j_{v}^{\mathrm{em}}(0)\right]\right| 0\right\rangle=\left(-g_{\mu v} q^{2}+q_{\mu} q_{v}\right) \Pi\left(-q^{2}\right),
$$

with the hadronic EM current $j_{\mu}^{\mathrm{em}}=\sum_{f} Q_{f} \bar{\psi}_{f} \gamma_{\mu} \psi_{f}$, and $Q_{f}$ being the EM charge of the quark $f$. The optical theorem relates the inclusive cross-section and thus the function $R(s)$ to the discontinuity of $\Pi$ in the complex plane

$$
R(s)=12 \pi \operatorname{Im} \Pi(-s-i \delta)
$$

The renormalization mode of the polarization operator $\Pi\left(Q^{2}\right)$ reads (see, e.g. [3])

$$
1+e^{2} \Pi\left(Q^{2} / \mu^{2}, \alpha_{s}\right)=Z_{3}^{\mathrm{ph}}+e^{2} \Pi_{0}\left(Q^{2}, \alpha_{s}^{0}\right),
$$

where $e=\sqrt{\alpha 4 \pi}$ and $\alpha$ is the (renormalazied) fine structure constant. Eq. (1.3) can be naturally deduced from the connection between $\Pi\left(Q^{2}\right)$ and the photon propagator $D_{\mu v}(q)$

$$
D_{\mu v}(q)=g_{\mu \nu} \frac{i}{q^{2}} \frac{1}{1+e^{2} \Pi\left(q^{2}=-Q^{2}\right)} .
$$


It is also convenient to introduce the Adler function as

$$
D\left(Q^{2}\right)=-12 \pi^{2} Q^{2} \frac{\mathrm{d}}{\mathrm{d} Q^{2}} \Pi\left(Q^{2}\right)=\int_{0}^{\infty} \frac{Q^{2} R(s) d s}{\left(s+Q^{2}\right)^{2}} .
$$

We define the perturbative expansions

$$
D\left(Q^{2}\right)=\sum_{i=0}^{\infty} d_{i} a_{s}^{i}\left(Q^{2}\right), R(s)=\sum_{i=0}^{\infty} r_{i} a_{s}^{i}(s)
$$

where $a_{s} \equiv \alpha_{s} / \pi$ and we have set the normalization scale $\mu^{2}=Q^{2}$ or o $\mu^{2}=s$ for the Euclidian and Minkowskian functions respectively. The results for generic values of $\mu$ can be easily recovered with standard RG techniques.

For the vector correlator the terms of order $a_{s}^{2}$ and $a_{s}^{3}$ have been evaluated nearly thirty and about fifteen years ago [13, 14], respectively. The $a_{s}^{4}$ corrections are conveniently classified according to their power of $n_{f}$, with $n_{f}$ denoting the number of light quarks. The $a_{s}^{4} n_{f}^{3}$ term is part of the "renormalon chain", the evaluation of the next term, of order $a_{s}^{4} n_{f}^{2}$, was a test case for the techniques used extensively in this paper and, furthermore, led to useful insights into the structure of the perturbative series already [15]. The rest: two remaining most difficult terms of orders $a_{s}^{4} n_{f}^{2}$ and $a_{s}^{4} n_{f}^{0}$ of equivalent complexity ${ }^{1}$ are not yet known. The results of their (partial) evaluation will be presented in the talk.

\section{Calculation of $d_{4}$ at $n_{f}=3$}

The complete five-loop calculation requires the evaluation of 2671 Abelian quenched plus about seventeen thousand non-abelian and non-quenched diagrams (see Fig 1).

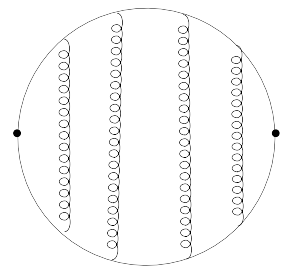

(a)

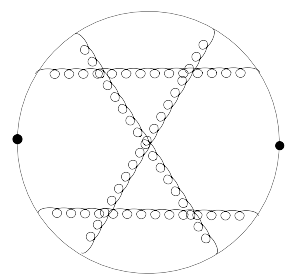

(b)

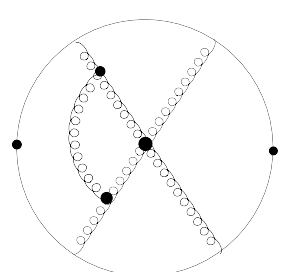

(c)

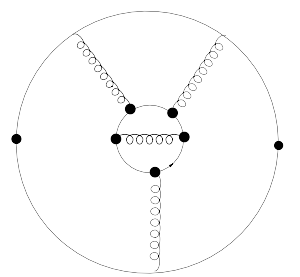

(d)

Figure 1: (a) (a),(b) Examples of five -loop quenched Abelian diagrams of order $a_{s}^{4} n_{f}^{0}$. (c) A five -loop non-abelian diagram. (d) A five -loop non-quenched abelian diagram of order $a_{s}^{4} n_{f}$

Using "infrared rearrangement" [16], the $R^{*}$ operation [17] and the prescriptions formulated in [18] to algorithmically resolve the necessary combinatorics, it is possible to express the absorptive part of the five-loop diagrams in terms of four-loop massless propagator integrals ("p-integrals"). Using then a representation for Feynman integrals proposed in $[19,20]$, these p-integrals can be reduced to a sum of 28 master integrals, with coefficients which are rational functions of the spacetime dimension $D$. These coefficients were, in a first step, evaluated in the large- $D$ limit, and,

\footnotetext{
${ }^{1}$ In the sense that they both contain all possible topologies.
} 
after evaluating sufficiently many terms of the $1 / D$ expansion, fully reconstructed [21]. This direct and largely automatic procedure required enormous computing resources and was performed on a 32+8-node SGI parallel computer with SMP architecture and on the HP XC4000 supercomputer of the federal state Baden-Württemberg using a parallel version [22] of FORM [23].

The construction of the large- $D$ limit requires in general huge storage resources, which naturally constrains the structure of the input p-integrals: they should better not contain any extra parameters like color coefficients, $n_{f}$-the number of light quark flavours contributing to internal fermion loops-, and so on. As a result we are forced to use a "slice" approach: that is to set all color coefficients to their numerical values and to fix $n_{f}$ to some integer. Thus, in order to compute the remaining two not-yet-known $\alpha_{s}^{4}$ contributions to $R(s)$ one should compute two slices: $n_{f}=0$ and $n_{f}=n_{0}$. Here $n_{0}$ stands for any non-zero integer which could be chosen at will. Since the evaluation of every single slice is a problem by itself, we decided to start from $n_{0}=3$ as the result has important physical applications for the analysis of the QCD corrections to hadronic $\tau$-decays (see, e.g. [4]).

Our result reads (we suppress the trivial factor $3 \sum_{f} Q_{f}^{2}$ throughout $^{2}$ )

$$
\begin{aligned}
d_{4}\left(n_{F}=3\right) & =\frac{78631453}{20736}-\frac{1704247}{432} \zeta_{3}+\frac{4185}{8} \zeta_{3}^{2}+\frac{34165}{96} \zeta_{5}-\frac{1995}{16} \zeta_{7} \\
& \approx 49.0757
\end{aligned}
$$

The corresponding expression for $R(s)$ is:

$$
1+a_{s}+1.6398 a_{s}^{2}+6.3710 a_{s}^{3}-106.8798 a_{s}^{4} .
$$

Since it will presumably take a long time until the next term of the perturbative series will be evaluated, it is of interest to investigate the predictive power of various optimization schemes empirically. Using the principles of "Fastest Apparent Convergence" (FAC) [24] or of "Minimal Sensitivity" (PMS) [25], which happen to coincide in this order, one gets [26, 27]

$$
d_{4}^{\text {pred }}\left(n_{f}=3\right)=27 \pm 16,
$$

with the central value of differing significantly from the exact result

$$
d_{4}^{\text {exact }}\left(n_{f}=3\right)=49.08 \text {. }
$$

However, within the error estimates [27], predicted and exact values are in agreement. The picture changes, once these estimates are used to predict the coefficient $r_{4}$. The prediction for the final result is significantly closer (in a relative sense) to the result of the exact calculation:

$$
r_{4}^{\text {pred }}\left(n_{f}=3\right)=-129 \pm 16, \quad r_{4}^{\text {exact }}\left(n_{f}=3\right)=-106.88
$$

This is in striking contrast to the case of the scalar correlator, where the predictions for the dynamical terms work well, but, as a consequence of the strong cancellations between dynamical and kinematical terms fail completely in the Minkowskian region [28].

\footnotetext{
${ }^{2}$ In this paper we present the results for the so-called "non-singlet" diagrams, where one and the same closed quark line is connected to the external currents. These are sufficient for a complete description of $\tau$-decays. For $e^{+} e^{-}$ annihilation they correspond to the dominant terms proportional $\sum_{f} Q_{f}^{2}$. The singlet contributions proportional $\left(\sum_{f} Q_{f}\right)^{2}$ arise for the first time in $\mathscr{O}\left(\alpha_{s}^{3}\right)$. They are known to be small, and will be evaluated at a later point.
} 


\section{Colour structure of $d_{4}$}

In general, it would be of great interest to know the $\alpha_{s}^{4}$ contribution for a generic color group. The structure of the result for, say, Adler function, may be predicted from [29]:

$$
d_{4}=\sum_{i=1,12} d_{4, i} c_{4, i}
$$

with the coefficients $d_{4, i}$ being polynomials in $\ln \left(\frac{\mu^{2}}{Q^{2}}\right)$ and the following colour factors read

$$
\begin{gathered}
c_{4}=\left\{d_{R} C_{F}^{4}, d_{R} C_{F}^{3} C_{A}, d_{R} C_{F}^{2} C_{A}^{2}, d_{R} C_{F} C_{A}^{3}, d_{R} C_{F}^{3} T_{F} n_{f}, d_{R} C_{F}^{2} C_{A} T_{F} n_{f}\right. \\
\left.d_{R} C_{F} C_{A}^{2} T_{F} n_{f}, d_{R} C_{F}^{2} T_{F}^{2} n_{f}^{2}, d_{R} C_{F} C_{A} T_{F}^{2} n_{f}^{2}, d_{R} C_{F} T_{F}^{3} n_{f}^{3}, d_{F}^{a b c d} d_{A}^{a b c d}, n_{f} d_{F}^{a b c d} d_{F}^{a b c d}\right\}
\end{gathered}
$$

Here $\left[T^{a} T^{a}\right]_{i j}=C_{F} \delta_{i j}$ and $f^{a c d} f^{b c d}=C_{A} \delta^{a b}$ are the quadratic Casimir operators of the fundamental and the adjoint representation of the Lie algebra and $\operatorname{tr}\left(T^{a} T^{b}\right)=T_{F} \delta^{a b}$ is the trace normalization of the fundamental representation. $d_{R}$ is the dimension of the fermion representation (i.e. the number of quark colours) and $n_{f}$ is the number of quark flavors. The exact definitions for the higher order group invariants $d_{F}^{a b c d} d_{A}^{a b c d}$ and $d_{F}^{a b c d} d_{F}^{a b c d}$ are given in [29]. For QCD with SU(3):

$$
C_{F}=4 / 3, C_{A}=3, T_{F}=1 / 2, d_{R}=3, d_{F}^{a b c d} d_{A}^{a b c d}=\frac{15}{2}, d_{F}^{a b c d} d_{F}^{a b c d}=\frac{5}{12} .
$$

We observe that to get the full colour structure of $d_{4}$ we should compute as many as eight extra slices (as four slices are already available: $d_{4,10}$ [30], $d_{4,9}$ and $d_{4,8}$ [15], as well as $S U(3)$ with $n_{f}=3$ ). Thus, complete evaluation of $d_{4}$ for generic colour group is a matter of future.

However, not all slices are equally difficult. For the case of the abelian U(1) group (QED!) the colour coefficients have especially simple form:

$$
C_{F}=1, C_{A}=0, T_{F}=1, d_{R}=1, d_{F}^{a b c d} d_{A}^{a b c d}=0, d_{F}^{a b c d} d_{F}^{a b c d}=1 .
$$

In addition the corresponding diagrams are somewhat simpler due to the absence of the three- and four-gluon couplings as well as any vertexes with ghost lines. An even simpler slice corresponds to case of quenched QED (qQED):

$$
C_{F}=1, C_{A}=0, T_{F}=1, d_{R}=1, n_{f}=0, d_{F}^{a b c d} d_{A}^{a b c d}=0, d_{F}^{a b c d} d_{F}^{a b c d}=0 .
$$

Thus, in general the quenched QED contribution to $d_{n}$ is equal to the coefficient in front of the $d_{R}\left(C_{F}\right)^{n}$ structure in the colour decomposition of $d_{n}$.

Up to order $\alpha^{3}$ the Adler function for qQED reads [14]

$$
D^{\mathrm{qQED}}(\alpha, Q)=1+3 A-\frac{3}{2} A^{2}-\frac{69}{2} A^{3}
$$

where $A=A(\mu)=\frac{\alpha(\mu)}{4 \pi}$. Note that $D^{\mathrm{qQED}}(\alpha, \mu / Q)$ does not depend on its second argument at all, which means that it is an essentially finite, scheme-independent quantity. It is instructive to compare eq. (3.3) to the $\beta$-function of $\mathrm{qQED}$, also known to the same order from [31, 32, 33]:

$$
\beta^{\mathrm{qQED}}=\frac{4}{3} A\left\{1+3 A-\frac{3}{2} A^{2}-\frac{69}{2} A^{3}\right\} .
$$


One observes the validity of the relation

$$
D^{\mathrm{qQED}}(\alpha, Q)=\frac{4}{3} A \beta^{\mathrm{qQED}}
$$

which can be easily proven in all orders of perturbation theory starting from (1.3). Thus, the evaluation of the five-loop contribution to $\beta^{\mathrm{qQED}}$ provides with us another slice, namely with $d_{4,1}$.

On the other hand, such a calculation is of importance by itself. Indeed, the $\beta$ - function of qQED possesses a number of remarkable and intriguing features.

- It is gauge and scheme independent in all orders.

- Its coefficients are simple rational numbers at three and four loops ${ }^{3}$.

- If $\beta^{\mathrm{qQED}}\left(\alpha_{0}\right)=0$ then $\alpha=\alpha_{0}$ leads to a self-consistent finite solution of (massless) QED $[34,35]$

A detailed knot-theoretic explanation of the rationality property at three loop level was given in $[36,37]$. At four loop the problem was thoroughly investigated with the help of a dimensionally background-field method in $[37,38]$. Some hope that the rationality property is not accidental but also holds in higher orders has been expressed in [38]. Unfortunately, no clear unambiguous prediction as for the structure of higher (five loops and beyond) orders has ever been made.

We have computed $d_{4,1}$. Our five loop result for the $\beta$ function reads:

$$
\beta^{\mathrm{qQED}}=\frac{4}{3} A+4 A^{2}-2 A^{3}-46 A^{4}+\left(\frac{4157}{6}+128 \zeta_{3}\right) A^{5} .
$$

Thus our calculation of $d_{4,1}$ has solved the problem: the rationality ceases to exist starting from fifth loop.

Acknowledgments. We thank David Broadhurst and Dirk Kreimer for useful discussions. This work was supported by the Deutsche Forschungsgemeinschaft in the Sonderforschungsbereich/Transregio SFB/TR-9 "Computational Particle Physics", by INTAS (grant 03-51-4007) and by RFBR (grant 05-02-17645). The computer calculations were partially performed on the HP XC4000 super computer of the federal state Baden-Württemberg at the High Performance Computing Center Stuttgart (HLRS) under the grant "ParFORM".

Note added. Recently we have finished the calculation of $d_{4}$ for a generic value of $n_{f}$. The reader is referred to [39] for details and for the discussion of phenomenological applications of the result.

\section{References}

[1] G. 't Hooft and M.J.G. Veltman, Nucl. Phys. B44 (1972) 189.

[2] G. 't Hooft, Nucl. Phys. B61 (1973) 455.

[3] K.G. Chetyrkin, J.H. Kühn and A. Kwiatkowski, Phys. Rep. 277 (1996) 189, and references therein.

[4] M. Davier, A. Hocker and Z. Zhang, Rev. Mod. Phys. 78 (2006) 1043, and references therein.

\footnotetext{
${ }^{3}$ The rationality at one and two loops takes place for any $\beta$-function irrespectively of the (massless) theory.
} 
[5] K.G. Chetyrkin, J.H. Kühn and M. Steinhauser, Phys. Lett. B371 (1996) 93, hep-ph/9511430.

[6] K.G. Chetyrkin, J.H. Kühn and M. Steinhauser, Nucl. Phys. B482 (1996) 213, hep-ph/9606230.

[7] K.G. Chetyrkin and J.H. Kühn, Phys. Lett. B248 (1990) 359.

[8] K.G. Chetyrkin and J.H. Kühn, Nucl. Phys. B432 (1994) 337, hep-ph/9406299.

[9] K.G. Chetyrkin, R.V. Harlander and J.H. Kühn, Nucl. Phys. B586 (2000) 56, hep-ph/0005139.

[10] P.A. Baikov, K.G. Chetyrkin and J.H. Kühn, Nucl. Phys. Proc. Suppl. 135 (2004) 243.

[11] A. Czarnecki and J.H. Kühn, Phys. Rev. Lett. 77 (1996) 3955, hep-ph/9608366.

[12] R. Harlander, T. Seidensticker and M. Steinhauser, Phys. Lett. B426 (1998) 125, hep-ph/9712228.

[13] K.G. Chetyrkin, A.L. Kataev and F.V. Tkachov, Phys. Lett. B85 (1979) 277.

[14] S.G. Gorishnii, A.L. Kataev and S.A. Larin, Phys. Lett. B259 (1991) 144.

[15] P.A. Baikov, K.G. Chetyrkin and J.H. Kühn, Phys. Rev. Lett. 88 (2002) 012001, hep-ph/0108197.

[16] A.A. Vladimirov, Theor. Math. Phys. 43 (1980) 417.

[17] K.G. Chetyrkin and V.A. Smirnov, Phys. Lett. B144 (1984) 419.

[18] K.G. Chetyrkin, Phys. Lett. B391 (1997) 402, hep-ph/9608480.

[19] P.A. Baikov, Phys. Lett. B385 (1996) 404, hep-ph/9603267.

[20] P.A. Baikov, Nucl. Instrum. Meth. A389 (1997) 347, hep-ph/9611449.

[21] P.A. Baikov, Phys. Lett. B634 (2006) 325, hep-ph/0507053.

[22] M. Tentyukov et al., (2004), cs.sc/0407066.

[23] J.A.M. Vermaseren, (2000), math-ph/0010025.

[24] G. Grunberg, Phys. Rev. D29 (1984) 2315.

[25] P.M. Stevenson, Phys. Rev. D23 (1981) 2916.

[26] A.L. Kataev and V.V. Starshenko, Mod. Phys. Lett. A10 (1995) 235, hep-ph/9502348.

[27] P.A. Baikov, K.G. Chetyrkin and J.H. Kühn, Phys. Rev. D67 (2003) 074026, hep-ph/0212299.

[28] P.A. Baikov, K.G. Chetyrkin and J.H. Kühn, Phys. Rev. Lett. 96 (2006) 012003, hep-ph/0511063.

[29] J.A.M. Vermaseren, S.A. Larin and T. van Ritbergen, Phys. Lett. B405 (1997) 327, hep-ph/9703284.

[30] M. Beneke, Nucl. Phys. B405 (1993) 424.

[31] J.L. Rosner, Phys. Rev. Lett. 17 (1966) 1190.

[32] J.L. Rosner, Annals Phys. 44 (1967) 11.

[33] S.G. Gorishnii et al., Phys. Lett. B256 (1991) 81.

[34] K. Johnson, R. Willey and M. Baker, Phys. Rev. 163 (1967) 1699.

[35] K. Johnson and M. Baker, Phys. Rev. D8 (1973) 1110.

[36] D.J. Broadhurst, R. Delbourgo and D. Kreimer, Phys. Lett. B366 (1996) 421, hep-ph/9509296.

[37] D.J. Broadhurst, Phys. Lett. B466 (1999) 319, hep-ph/9909336.

[38] D.J. Broadhurst, (1999), hep-th/9909185.

[39] P.A. Baikov, K.G. Chetyrkin and J.H. Kühn, (2008), arXiv:0801.1821 [hep-ph]. 